19

\title{
Нелинейное поглощение растворов метиленового голубого в присутствии плазмонных наночастиц с различным поверхностным зарядом
}

\author{
(C) А.П. Русинов, М.Г. Кучеренко \\ Оренбургский государственный университет, \\ 460018 Оренбург, Россия \\ e-mail: sano232@mail.ru
}

Поступила в редакцию 15.03.2020 г.

В окончательной редакции 15.05.2020 г.

Принята к публикации 20.05.2020 г.

\begin{abstract}
Методом Z-сканирования при непрерывной накачке исследовано нелинейное поглощение света водных и спиртовых растворов метиленового голубого. Показано, что механизмы оптической нелинейности данных растворов меняются в зависимости от концентрации красителя и вида растворителя. Выявлена зависимость амплитуды нелинейного поглощения молекул красителя от концентрации и знака поверхностного заряда наночастиц золота и серебрав растворе. Рассмотрены математические модели оптической нелинейности для концентрированных и разбавленных растворов молекул красителя, в последнем случае учтено влияние плазмонных наночастиц на нелинейно-оптические характеристики растворов метиленового голубого.
\end{abstract}

Ключевые слова: нелинейное поглощение света, метод Z-сканирования, метиленовый голубой, плазмонные наночастицы.

DOI: $10.21883 /$ OS.2020.09.49880.92-20

\section{Введение}

Нелинейные свойства оптических материалов, связанные с некогерентным насыщением поглощения, многофотонными процессами, тепловой или керровской нелинейностью представляют несомненный интерес для современной физики и ее приложений. В прикладном смысле материалы с высокими оптическими нелинейностями привлекательны точки с точки зрения их использования в качестве оптических ограничителей, оптических переключателей, систем управления формой и длительностью лазерных импульсов и т.д.

В качестве нелинейно-оптических сред широко используются жидкие растворы, содержащие фотоактивные центры. По сравнению, например, с кристаллическими средами они более универсальны, параметры таких систем могут варьироваться в широчайших пределах за счет выбора состава. В качестве фотоактивных компонентов растворов традиционно используются молекулы органических красителей, часто в этой роли выступают наночастицы (НЧ) металлов, квантовые точки, углеродные НЧ и их композиции.

Среди широкого перечня экспериментальных методов исследования нелинейно-оптических свойств различных систем [1-4], в последнее время большое развитие получил метод Z-сканирования из-за сравнительной простоты его реализации и интерпретации результатов. Метод заключается визмерении интенсивности прошедшего через образецсфокусированного светового потока, при различных положениях образца относительно фокуса пучка. Это позволяет в режиме открытой апертуры измерять зависимость коэффициента нелинейного поглощения образца от плотности мощности падающего излучения, а в режиме ограниченной (закрытой) апертуры определять нелинейную рефракцию света в материале [3].

\section{Материалы и методы}

Как правило, в качестве источников излучения в установках Z-сканирования используются импульсные лазеры, позволяющие получать в перетяжке пучка высокую плотность мощности, достаточную для исследования нелинейных процессов в большинстве сред. При использовании непрерывных лазеров плотность мощности светового потока в фокусе существенно меньше, что сужает применимость метода, однако для изучения некоторых аспектов оптической нелинейности растворов красителей данной мощности вполне достаточно. В нашем случае установка Z-сканирования реализована на основе непрерывного полупроводникового лазера с мощностью $400 \mathrm{~mW}$ и длиной волны $660 \mathrm{~nm}$. Оптическая схема установки показана на рис. 1 , радиус перетяжки пучка накачки в фокальной плоскости линзы составлял $20 \mu \mathrm{m}$, рэлеевская длина перетяжки пучка $z_{R}=2 \mathrm{~mm}$. Толщина кюветы для исследуемых образцов - $1 \mathrm{~mm}$, для ее перемещения вдоль перетяжки пучка накачки использовался шаговый двигатель с максимальной точностью позиционирования $0.05 \mathrm{~mm} /$ point. Нелинейное поглощение растворов измерялось в режиме открытой апертуры.

Для оптической сенсибилизации исследуемых водных и водно-спиртовых растворов в данной работе использовался органический краситель - метиленовый 


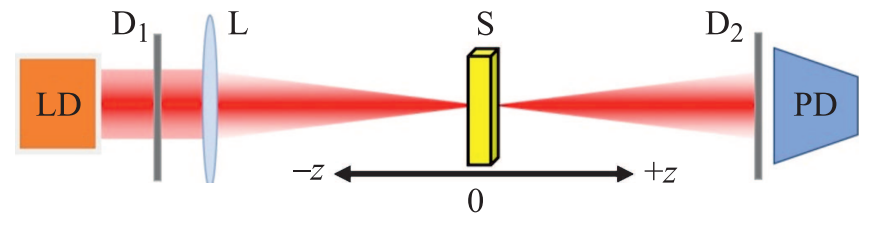

Рис. 1. Схема Z-сканирования. $z-$ положение образца относительно фокуса линзы. LD - источник излучения, $\mathrm{D}_{1}$, $\mathrm{D}_{2}$ - диафрагмы; L - линза, $\mathrm{PD}$ - фотодетектор, $\mathrm{S}-$ образец.
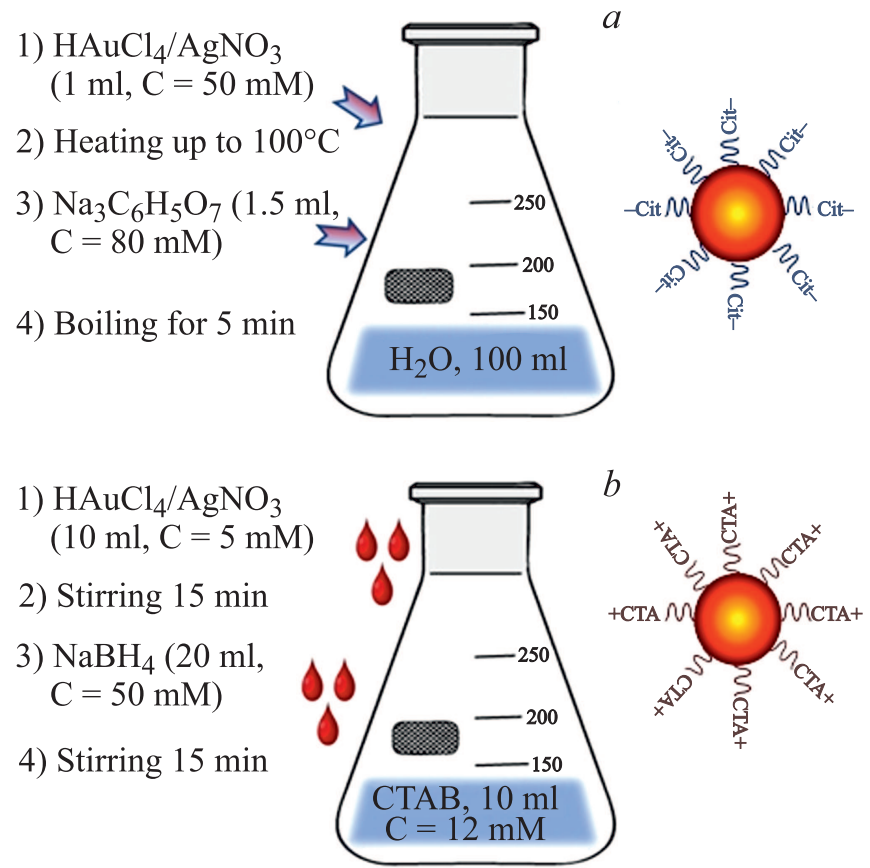

Рис. 2. Схема синтеза НЧ золота и серебра цитратным $(a)$ и боргидридным $(b)$ восстановлением.

голубой (МГ) в концентрациях от $10^{-5}$ до $10^{-3} \mathrm{M}$. Выбор красителя обусловлен его широким применением в оптических и фотонных устройствах, био- и медицинских приложениях [5-7]. Вводных и водно-спиртовых растворах данный краситель заряжен положительно.

Синтез отрицательно заряженных НЧ золота и серебра был осуществлен по методу Туркевича-Френса $[8,9]$ (рис. $2, a$ ). В данном методе восстановителем металла и стабилизатором поверхности НЧ выступал цитрат натрия, а в качестве источников атомов золота использовался тетрахлораурат водорода (в параллельной методике синтеза источником атомов серебра выступал $\mathrm{AgNO}_{3}$ ). Преимущества метода заключаются в относительно узком распределении по размерам синтезированных частиц, порядка $20 \mathrm{~nm}$, интенсивной полосе поглощения в водных растворах на $520 \mathrm{~nm}$ для золота и $420 \mathrm{~nm}$ для серебра, а также в способности относительно легко агрегировать в присутствии биополимерных соединений.
Для осуществления синтеза в стеклянную колбу, помещенную на нагреватель MR Hei-Standard с магнитной мешалкой, вносили $100 \mathrm{ml}$ деионизованной воды и $1 \mathrm{ml} 50 \mathrm{mM}$ водного раствора $\mathrm{HAuCl}_{4}$ (или $\mathrm{AgNO}_{3}$ ) и доводили до кипения. Затем, при перемешивании, добавляли к горячему раствору $1.5 \mathrm{ml} 80 \mathrm{mM}$ раствора цитрата натрия. После того как раствор изменял цвет, его кипятили в течение нескольких минут до появления устойчивой рубиновой (для НЧАu) или опалесцирующежелтой (для $\mathrm{HЧAg}$ ) окраски. Затем смесь охлаждали при перемешивании. Полученный раствор выдерживали в темном месте в течение $24 \mathrm{~h}$ для полной стабилизации НЧ и завершения процессов перекристаллизации. Концентрация НЧ, синтезированных таким образом, равнялась $120 \mu \mathrm{g} / \mathrm{ml}$ (или $0.6 \mathrm{mM}$ ). Согласно [10] данные НЧ заряжены отрицательно, измеренный методом динамического рассеяния света $\xi$-потенциал равен $-18 \mathrm{mV}$.

Синтез НЧ золота, стабилизированных ЦТМА, осуществляли по боргидридной методике, подобно описанной в $[10,11]$ (рис. $2, b$ ). В данной методике в качестве восстановителя использовался боргидрид натрия, в качестве стабилизатора - ЦТМА, источником атомов золота также выступал тетрахлораурат водорода (в параллельной методике синтеза источником атомов серебра $\left.-\mathrm{AgNO}_{3}\right)$.

Для осуществления синтеза в стеклянную колбу, помещенную на нагреватель MR Hei-Standard с магнитной мешалкой, вносили $10 \mathrm{ml} 12 \mathrm{mM}$ раствора бромида ЦТMA, затем по каплям, при интенсивном перемешивании, добавляли $10 \mathrm{ml} 5 \mathrm{mM}$ раствора $\mathrm{HAuCl}_{4}$ (или $\mathrm{AgNO}_{3}$ ), полученный раствор перемешивали $15 \mathrm{~min}$. Затем по каплям при перемешивании добавляли $20 \mathrm{ml} 50 \mathrm{mM}$ раствора $\mathrm{NaBH}_{4}$. После перемешивания в течение $30 \mathrm{~min}$ раствор также выдерживали $24 \mathrm{~h}$ в темноте.

Концентрация НЧ золота, синтезированных таким образом, равна $140 \mu \mathrm{g} / \mathrm{ml}$ ( $0.7 \mathrm{mM}$ по золоту). Согласно [10], данные НЧ заряжены положительно, измеренный методом динамического рассеяния света $\xi$ потенциал равен $+40 \mathrm{mV}$. Спектры поглощения НЧ серебра имеют плазмонный пик на $435 \mathrm{~nm}$, у золотых НЧ максимум поглощения расположен вблизи $525 \mathrm{~nm}$. Максимумы спектров поглощения золотых НЧ, полученных по разным методикам, достаточно близки и незначительно отличаются только амплитудой и шириной полосы плазмонного резонанса. Отметим здесь, что плазмонные полосы НЧ золота и серебра слабо перекрываются со спектральным диапазоном лазера накачки установки Zсканирования $(660 \mathrm{~nm})$, т.е. влияние НЧ на нелинейные свойства образцов имеет опосредованный характер вследствие их взаимодействия с молекулами красителя.

Средний радиус НЧ серебра составляет $15 \mathrm{~nm}, \mathrm{HЧ} \mathrm{зо-}$ лота, полученные цитратным восстановлением, крупнее, их средний радиус $-40 \mathrm{~nm}$. Золотые НЧ синтезированные по боргидридной методике имеют более широкий разброс по размерам (сравнительно с цитратным методом) и средний радиус около $10 \mathrm{~nm}$. 

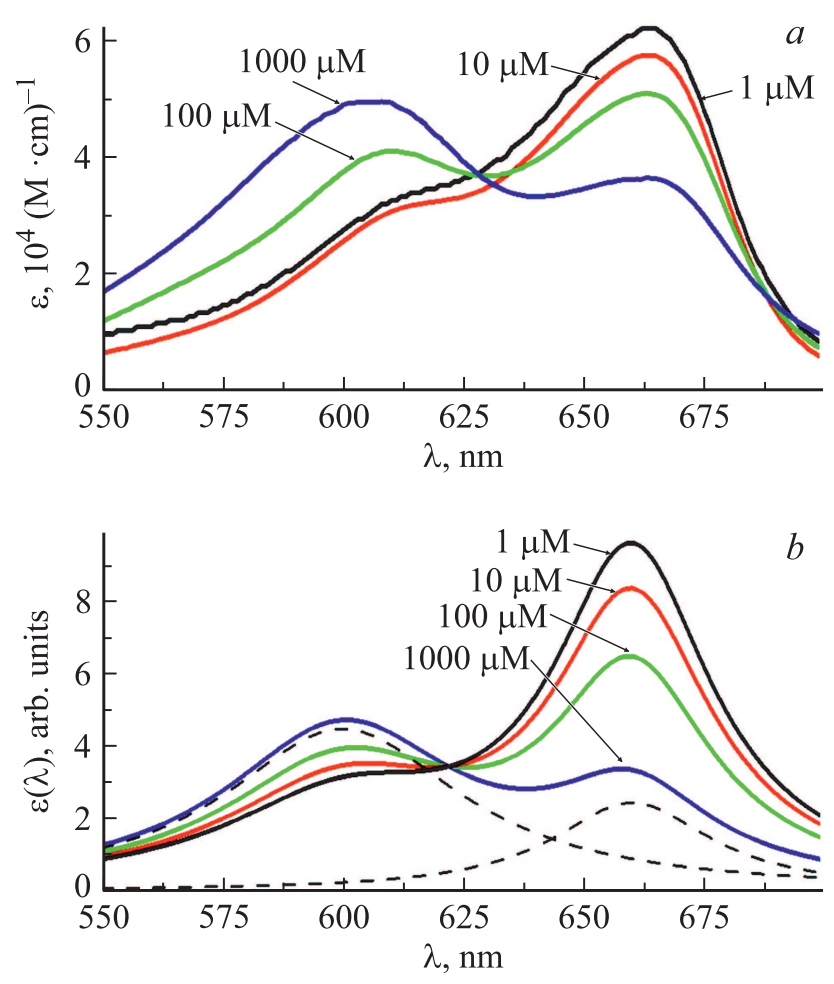

Рис. 3. Спектры поглощения растворов МГ при различных концентрациях: $a-$ эксперимент, $b-$ модельный расчет.

\section{Нелинейное поглощение концентрированных растворов красителя}

При изменении концентрации МГ в водных растворах существенно изменяется спектр поглощения системы. На рис. 3 видно, что с увеличением концентрации красителя поглощение в полосе 650-670 nm уменьшается, а в полосе 590-610 nm - растет. Более длинноволновая полоса отвечает мономерной форме МГ, а коротковолновая - связана с образованием комплексов (димеров, тримеров и пр.). Нелинейное поглощение данных растворов,измеренное методом Z-сканирования в режиме открытой апертуры,также существенно и немонотонно зависит от концентрации МГ (рис. 4,a,c). Так, при малых концентрациях красителя пропускание раствора в области фокуса пучка накачки увеличивается, что говорит об оптическом просветлении. С увеличением концентрации красителя амплитуда просветления возрастает, достигая максимальной относительной величины в 5\% при концентрации красителя порядка $10^{-4} \mathrm{M}$.

При дальнейшем увеличении концентрации красителя сигнал Z-сканирования в области перетяжки пучка накачки начинает уменьшаться, т. е. изменяется знак нелинейности, вместо нелинейного просветления проявляет себя нелинейное усиление поглощения образца. Для концентрации МГ свыш $10^{-3} \mathrm{M}$ амплитуда данного эффекта, монотонно возрастая, достигает значений в сотни процентов. Также с ростом концентрации МГ имеет
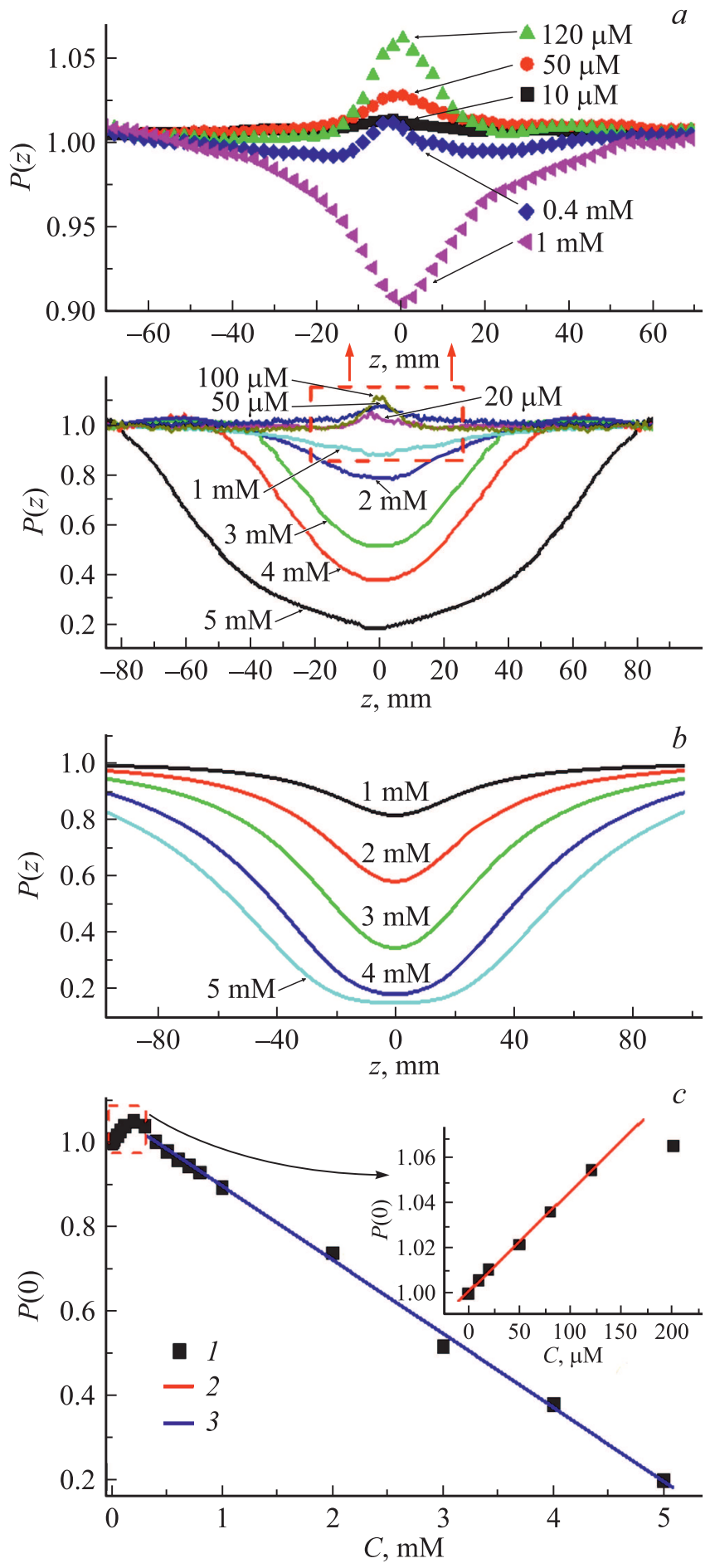

Рис. 4. Сигналы Z-сканирования конфентрированных водных растворов МГ при различных концентрациях красителя: $a-$ эксперимент, $b-$ модельный расчет, $c-$ амплитуда сигнала в перетяжке пучка.

место расширение пространственной области нелинейного усиления поглощения, что связано с уменьшением порога и изменением механизма нелинейности раствора.

Из рис. 3 можно отметить, что пороговая концентрация $10^{-4} \mathrm{M}$, отвечающая изменению механизма нелиней- 
ности раствора, также связана с усилением агрегации молекул красителя в димерные и тримерные формы.

В рамках упрощенной качественной модели общая концентрация молекул красителя может быть представлена в виде суммы его мономерной и димерной форм: $n_{\mathrm{All}}=n_{\text {Mon }}+2 n_{\text {Dim. }}$. Концентрация $n_{\text {Dim }}$ при этом определяется коэффициентом димеризации $k=n_{\mathrm{Dim}} / n_{\mathrm{All}}=f\left(n_{\mathrm{All}}\right)$, который сам является функцией общей концентрации молекул [12]. Представляя спектр поглощения образца в виде суммы мономерных и димерных полос аппроксимированных лоренцевыми функциями, можно получить хорошее согласие модельных кривых (рис. $3, b)$ и экспериментальных спектров поглощения (рис. $3, a)$.

Наблюдаемый механизм нелинейного усиления поглощения концентрированных растворов можно пояснить следующим: агрегированные молекулы взаимодействуют с излучением накачки $(\lambda=660 \mathrm{~nm})$ существенно слабее мономеров красителя, однаков области перетяжки пучка, при большой плотности мощности излучения, агрегаты разрушаются, это ведет к увеличению концентрации мономеров и, следовательно, к усилению поглощения раствора. Математически это можно представить в виде зависимости коэффициента димеризации от интенсивности лазерного пучка:

$$
k\left(n_{\mathrm{All}}, I(z)\right)=A \ln \left(n_{\mathrm{All}}^{-1}\right)\left(1+I(z) / I_{S}\right)^{-1} .
$$

Здесь $A$ и $I_{S}$ - некоторые модельные параметры, интенсивность пучка накачки

$$
I(z)=I_{0}\left(1+\frac{z^{2}}{z_{R}^{2}}\right)^{-1},
$$

где $z_{R}=\pi w_{0}^{2} / \lambda-$ рэлеевская длина каустики гауссова пучка, $\lambda$ - длина волны, $w_{0}$ - радиус и $I_{0}-$ интенсивность поля в перетяжке пучка отвечающие координате $z=0$.

На рис. 4, $b$ показано качественное соответствие расчетных и экспериментальных спектров Z-сканирования в концентрированных растворах МГ.

Аналогичные измерения для спиртовых растворов МГ и растворов с добавлением плазмонных НЧ показывают, что при больших концентрациях красителя рассмотренный выше механизм нелинейного поглощения является преобладающим, и практически не зависит от вида растворителя или наличия иных компонентов в растворе.

\section{Нелинейное поглощение разбавленных растворов красителя}

В данной части работы исследовались растворы красителя с концентрацией менее $10^{-4} \mathrm{M}$. При данных концентрациях агрегация выражена слабо и нелинейность растворов определяется процессами в молекулах МГ в мономерной форме. В этом случае сигналы Z-сканирования образцов обнаруживают сильную зависимость от вида растворителя.
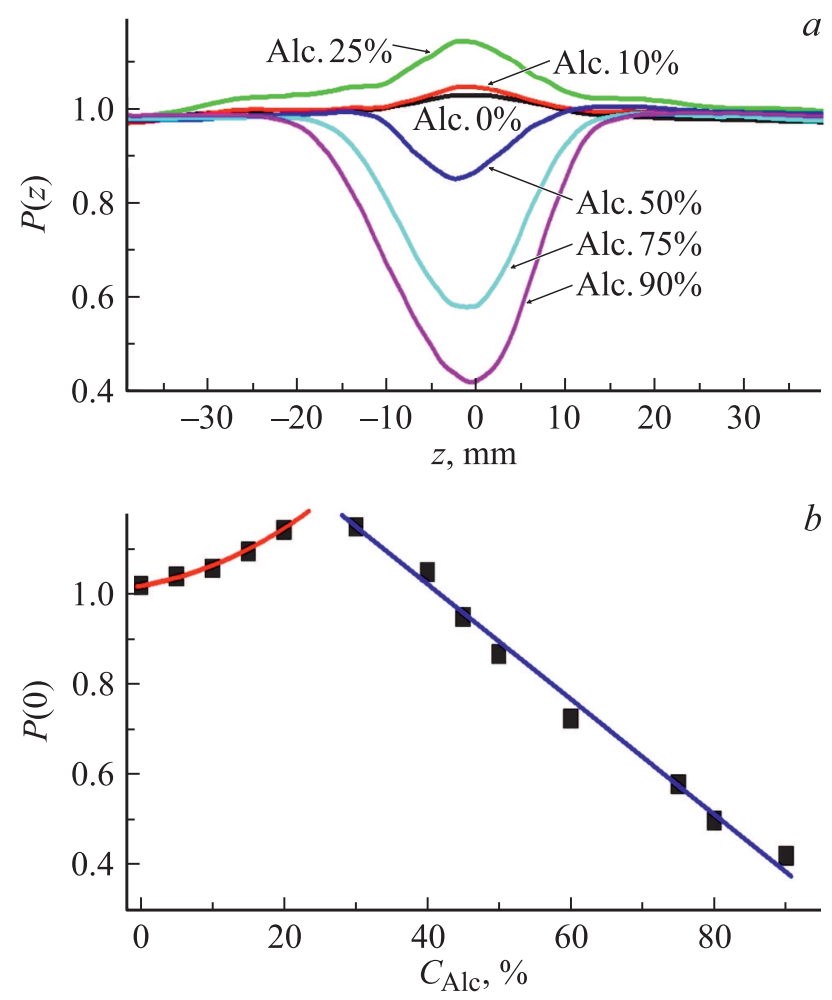

Рис. 5. Сигналы Z-сканирования с открытой апертурой водноспиртовых растворов МГ с концентрацией $5 \cdot 10^{-5} \mathrm{M}$ при различных соотношениях этанол-вода.

Из рис. 5, $a, b$ следует, что в водно-этанольном раствоpe, при неизменной концентрации красителя, увеличение доли спирта в диапазоне от 0 до 25\% приводит к усилению насыщения поглощения образцов (увеличению мощности проходящего пучка в перетяжке в 1.1 раза). Дальнейшее увеличение доли спирта в растворе (50\% и более) изменяет вид сигналов Z-сканирования, в перетяжке наблюдается существенное ослабление сигнала, т.е. в растворе наводится нелинейное усиление поглощения (амплитуда данного эффекта превышает 50\%).

Считая скорость перемещения образца вдоль оси пучка (скорость изменения интенсивности) малой по сравнению с характерными временами переходных процессов в среде, механизмы нелинейного поглощения разбавленных водно-спиртовых растворов МГ можно рассмотреть на основе анализа населённостей различных энергетических состояний поглощающих центров при постоянной накачке. Энергетическая структура молекул МГ может быть представлена 5-уровневой схемой (рис. 6), где обозначены через $S_{0}$ - основное состояние молекулы, $S_{1}$ и $S_{2}-$ нижнее и вышележащие по энергии синглетные, $T_{1}$ и $T_{2}$ нижнее и вышележащие по энергии триплетные возбужденные энергетические состояния соответственно. На длине волны накачки $660 \mathrm{~nm}$ $(h v \sim 2 \mathrm{eV})$ наиболее интенсивными являются индуцированные переходы $S_{0} \rightarrow S_{1}$ и $T_{1} \rightarrow T_{1}$ с сечениями переходов [7] $\sigma_{01} \sim \sigma_{12}^{*} \sim 10^{-15} \mathrm{~cm}^{-2}$. Индуцирован- 


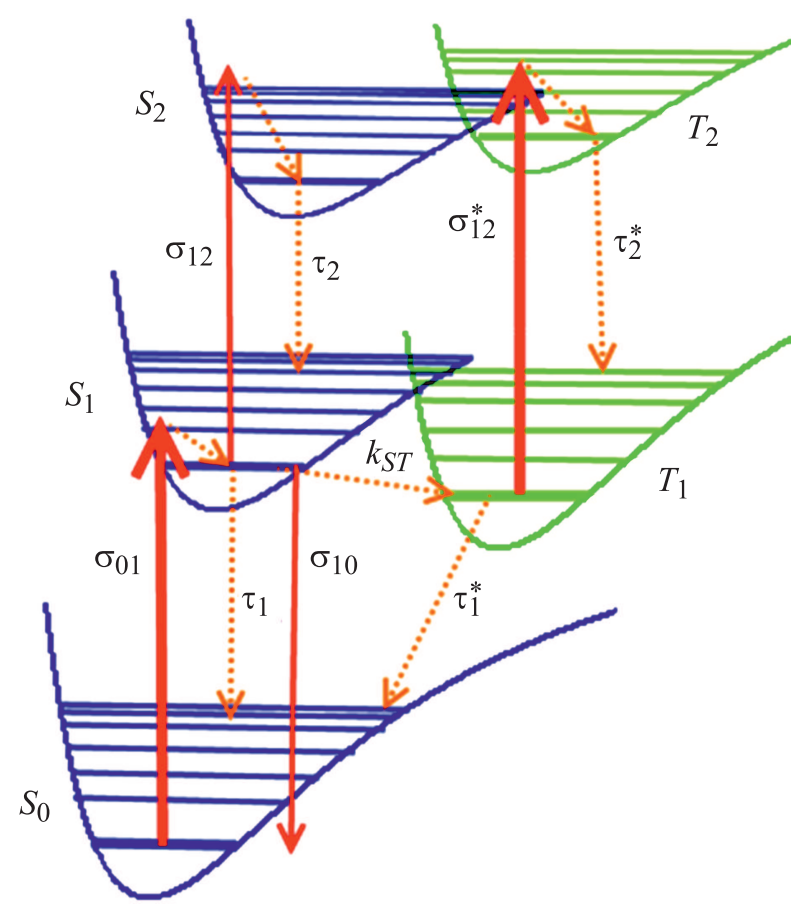

Рис. 6. Схема энергетических переходов в молекуле МГ.

ные переходы $S_{1} \rightarrow S_{0}$ и $S_{1} \rightarrow S_{2}$ имеют существенно меньшую интенсивность, так как населенность уровня $S_{1}$ сравнительно невысока и малы сечения переходов $\sigma_{12} \sim \sigma_{12} \sim 10^{-17} \mathrm{~cm}^{-2}$. Характерные времена релаксации уровня $S_{1}$ имеют порядок $\tau_{1} \sim k_{\mathrm{ST}}^{-1} \sim 10^{-9} \mathrm{~s}$, а $\tau_{1}^{*}-$ время релаксации триплетного уровня $T_{1}$ существенно больше.

Учитывая высокую скорость дезактивации состояний $S_{2}$ и $T_{2}$ (порядка $10^{13}-10^{14} \mathrm{~s}^{-1}$ ) можно считать их населенность близкой к нулю и, таким образом, ограничится рассмотрением трехуровневой схемы. Система балансных кинетических уравнений для населенностей может быть записана в виде

$$
\left\{\begin{array}{l}
\frac{\partial n_{S 0}}{\partial t}=-\left[\sigma_{01} n_{S 0}(t)-\sigma_{10} n_{S 1}(t)\right] I+\frac{1}{\tau_{1}} n_{S 1}(t)+\frac{1}{\tau_{1}^{*}} n_{T 1}(t), \\
\frac{\partial n_{S 1}}{\partial t}=\left[\sigma_{01} n_{S 0}(t)-\sigma_{10} n_{S 1}(t)\right] I-\frac{1}{\tau_{1}} n_{S 1}(t)-k_{S T} n_{S 1}(t), \\
\frac{\partial n_{T 1}}{\partial t}=k_{S T} n_{S 1}(t)-\frac{1}{\tau_{1}^{*}} n_{T 1}(t),
\end{array}\right.
$$

где $I$ - интенсивность (плотность потока фотонов) пучка накачки, параметрически зависящая от $z$ и измеряемая в photon $/ \mathrm{cm}^{2}$. В любой момент времени $t$ выполняется условие интегрального баланca $n_{S 0}(t)+n_{S 1}(t)+n_{T 1}(t)=n \quad(n=\mathrm{const})$, отражающее факт отсутствия необратимого расхода реагентов.

Так как скорость изменения интенсивности пучка при Z-сканировании существенно ниже скоростей перераспределения населенностей уровней молекул красителя, решение данной системы можно выполнить в квазистационарном приближении, при $t \rightarrow \infty$ [13]. Тогда коэффициент поглощения, с учетом переходов на высо- ковозбужденные уровни $S_{2}$ и $T_{2}$ определяется как

$$
\alpha(I)=\left(\sigma_{01} n_{S 0}(I)-\sigma_{10} n_{S 1}(I)\right)+\sigma_{12} n_{S 1}(I)+\sigma_{12}^{*} n_{T 1}(I) .
$$

Здесь первые два слагаемых описывают некогерентное насыщение поглощения в переходе между $S_{0^{-}}$и $S_{1^{-}}$ состояниями,

$$
\alpha_{\text {Sat }}(I)=\sigma_{01} n_{S 0}(I)-\sigma_{10} n_{S 1}(I)=\frac{\alpha_{0}}{1+I / I_{S}},
$$

где $\alpha_{0}=\sigma_{01} n-$ линейный коэффициент поглощения,

$$
I_{S}=\frac{\tau_{1}^{-1}+k_{S T}}{\sigma_{01}\left(1+k_{S T} \tau_{1}^{*}\right)+\sigma_{10}}
$$

- интенсивность насыщения раствора.

Из рис. 7, $a, b$ следует, что для водных растворов МГ характерен именно этот механизм нелинейности, что говорит о малом накоплении молекул красителя в триплетном состоянии.

В водно-этанольных растворах с увеличением доли спирта наблюдается нелинейное усиление поглощения, что связано с увеличением времени жизни возбужденных состояний молекул МГ в спиртовых растворах и,

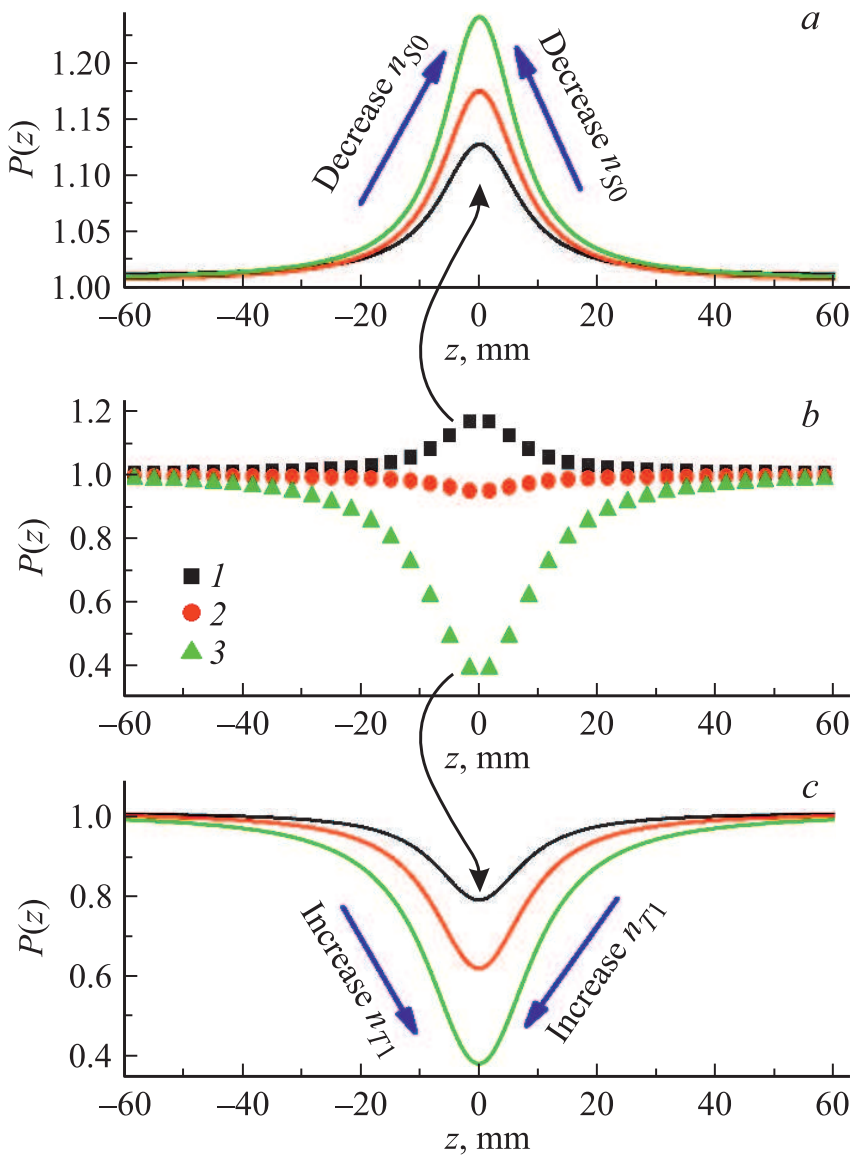

Рис. 7. Нелинейное поглощение раствора молекул красителя $(b)$ на уровнях $1-S_{0}, 2-S_{1}, 3-T_{1} ; a-$ оптическое просветление $S_{0}$ состояния; $c-$ наведенное поглощение состояния $T_{1}$. 
следовательно, с увеличением их заселенности раствоpax (рис. 7,b,c). Так как время жизни триплетного возбужденного состояния $T_{1}$ много больше, чем синглетного $-S_{1}$, то главную часть наведенного дополнительного поглощения будет давать четвертое слагаемое

$$
\alpha_{\text {Add }}(I)=\sigma_{12}^{*} n_{T 1}(I)=\frac{\beta I}{1+I / I_{S}}
$$

где

$$
\beta=\frac{\sigma_{12}^{*} \tau_{1}^{*}}{1+\left(k_{S T} \tau_{1}\right)^{-1}}
$$

\section{Влияние плазмонных НЧ на нелинейное поглощение красителя}

Для определения влияния НЧ золота и серебра на нелинейное поглощение МГ были приготовлены серии растворов в воде и в этаноле с постоянной концентрацией молекул красителя $\left(C=5 \cdot 10^{-5} \mathrm{M}\right)$ и различным содержанием плазмонных НЧ $(0,20,40,60,80 \%)$, где $100 \%$ содержание НЧ в растворах соответствует концентрации $0.7 \mathrm{mM}$.

Из рис. 8, $а$ следует, что в водных растворах МГ добавление НЧ, стабилизированных цитрат-ионом и имеющих отрицательный поверхностный заряд, приводит к уменьшению нелинейного насыщения поглощения красителя. При этом, если для НЧ золота максимальное уменьшение сигнала составляет около $20 \%$, то для
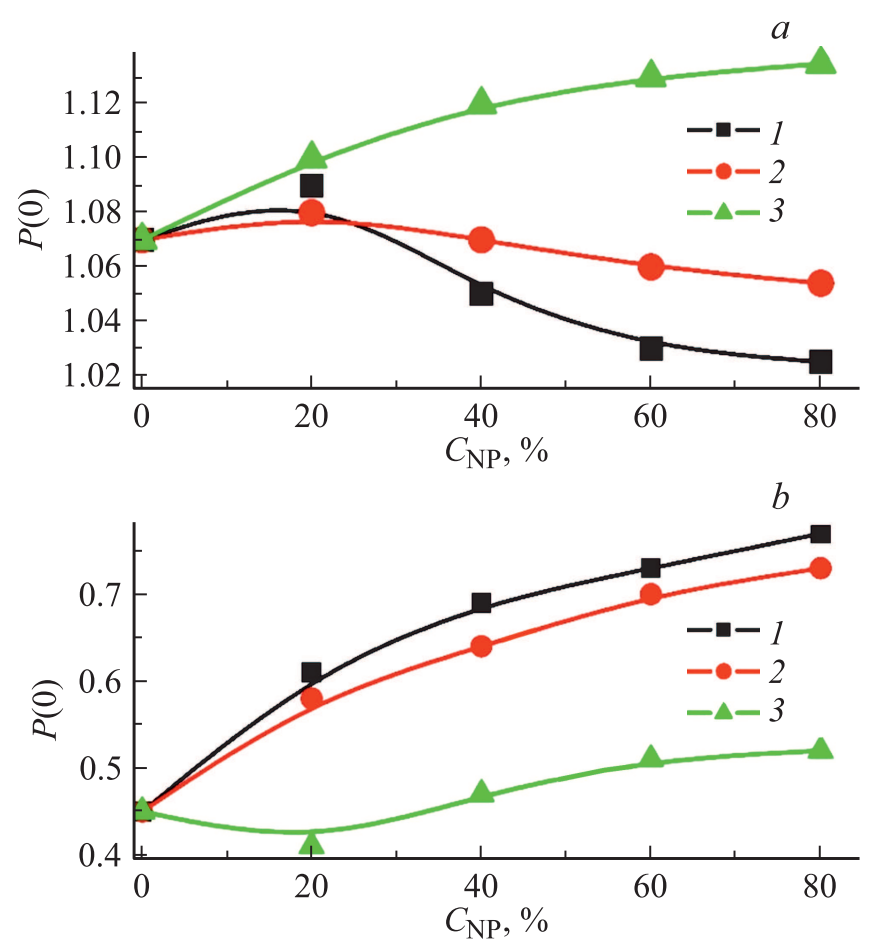

Рис. 8. Сигналы Z-сканирования водных этанольных растворов МГ с концентрацией $5 \cdot 10^{-5} \mathrm{M}$ при различных концентрациях НЧ. 1 - золотые; 2 - серебряные НЧ, приготовленные цитратным методом; 3 - золотые НЧ, приготовленные боргидридным методом.

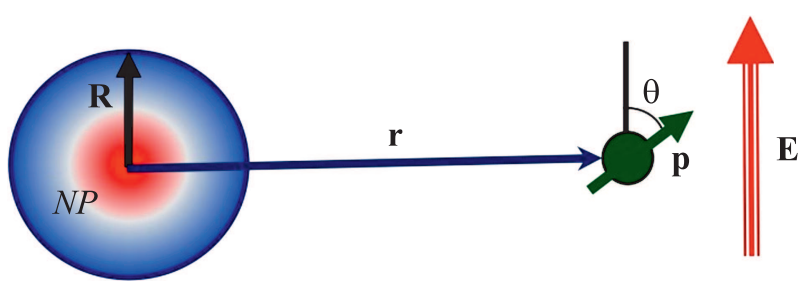

Рис. 9. Геометрия системы „НЧ-молекула-поле“.

НЧ серебра - уже 60\%. Положительно заряженные НЧ, приготовленные боргидридным восстановлением, увеличивают амплитуду нелинейного оптического просветления раствора практически вдвое.

В спиртовых растворах (рис. $8, b$ ) внесение отрицательно заряженных НЧ также приводит к уменьшению наведенного нелинейного поглощения красителя практически в 3 раза, однако существенных различий между НЧ серебра и золота при этом не наблюдается. Для положительно заряженных НЧ (боргидридная методика) данная зависимость практически отсутствует.

В рамках квазистатического приближения [14,15] можно показать, что вблизи плазмонной НЧ вероятность переходов между энергетическими состояниями молекулы красителя определяется суммарным дипольным моментом системы „НЧ-молекула“ (рис. 9)

$$
\mathbf{p}^{\prime}=[\overleftrightarrow{\mathbf{I}}+\overleftrightarrow{\mathbf{G}}(\mathbf{r}) \overleftrightarrow{\alpha}(\omega)] \mathbf{p}
$$

где $\overleftrightarrow{\mathbf{I}}$ - единичная матрица, p - дипольный момент перехода в молекуле,

$$
\overleftrightarrow{\mathbf{G}}(\mathbf{r})=-\frac{1}{r^{3}}\left(\overleftrightarrow{\mathbf{I}}-3 \frac{\mathbf{r} \otimes \mathbf{r}}{r^{2}}\right)
$$

— функция Грина точечного дипольного источника,

$$
\alpha(\omega)=\frac{\varepsilon(\omega)-\varepsilon_{m}}{\varepsilon(\omega)+2 \varepsilon_{m}} R^{3}
$$

- тензор поляризуемости НЧ, для сферической частицы сводящийся к скалярной величине, $R$ - радиус НЧ, $\varepsilon_{m}$ - диэлектрическая проницаемость среды и $\varepsilon(\omega)-$ диэлектрическая проницаемостьметалла, которая может быть определена как исходя из теоретическихмоделей, так и измерена экспериментально.

Тогда вероятности индуцированных переходов в объединенной системе определяются как

$$
w_{\text {ind }}(\omega)=2 \frac{\left|\mathbf{p}^{\prime} \mathbf{E}_{0}\right|^{2}}{\hbar^{2}} .
$$

Для геометрии системы „НЧ-молекула-поле“, представленной на рис. 9 вероятность индуцированных переходов может быть записана как

$$
\begin{aligned}
& w_{\text {ind }}(\omega)=\left(\frac{\mathbf{p} \mathbf{E}_{0}}{\hbar}\right)^{2}\left(1+\frac{\operatorname{Re} \alpha(\omega)}{r^{3}}(1+3 \cos 2 \theta)\right. \\
& \left.+\frac{|\alpha(\omega)|^{2}}{4 r^{6}}(1+3 \cos 2 \theta)^{2}\right)
\end{aligned}
$$


Здесь первое слагаемое в скобках отвечает за переходы в уединенной молекуле, а второе и третье описывают изменение вероятности переходов в присутствии НЧ. При этом второе - интерференционное слагаемое более ,дальнодействующее“ по сравнению с третьим и, в отличие от него, может приводить как к усилению, так и к уменьшению $w_{\text {ind }}(\omega)$ - в зависимости от знака этого слагаемого. Таким образом, влияние НЧ на индуцированные процессы в молекуле красителя в первом приближениии можно свести к изменению сечения поглощения для перехода между $i$ и $j$ состояниями:

$$
\sigma_{i j}^{\mathrm{ef}}=\sigma_{i j}+\sigma_{i j}^{\mathrm{NP}} .
$$

Помимо рассмотренных выше процессов также необходимо учитывать безызлучательный перенос энергии электронного возбуждения молекул на плазмонные моды НЧ с их последующим затуханием. Как показано в [16], скорость таких переходов определяется как

$$
U_{r l}(\omega)=\frac{1}{2 \hbar} \frac{p^{2}}{\varepsilon_{m}^{2} r^{6}}\left(1+3 \cos ^{2} \theta\right) \operatorname{Im} \alpha(\omega)
$$

и проявляет себя, главным образом, на достаточно малых расстояниях для тесных пар „НЧ-молекула“. Качественно данный процесс приводит к ускорению дезактивации возбужденных состояний и может быть учтен через уменьшение времени жизни $i$ уровня в молекуле красителя:

$$
\frac{1}{\tau_{i}^{\text {ef }}}=\frac{1}{\tau_{i}}+\frac{1}{\tau_{i}^{\mathrm{NP}}}
$$

Данный подход позволяет объяснить различное влияние плазмонных НЧ с противоположным знаком поверхностного заряда на оптическую нелинейность МГ. Так, в водных растворах наблюдается оптическое насыщение поглощения, связанное с уменьшением населенности основного состояния $S_{0}$. НЧ, имеющие отрицательный заряд (взаимодействуют с положительно заряженными молекулами МГ, что приводит к их сближению и образованию „тесных“ комплексов „краситель-НЧ“. На таких расстояниях сильнее проявляется безызлучательное тушение возбужденых молекул красителя НЧ, населенность основного уровня увеличивается и оптическое просветление растворов уменьшается. Относительно положительно заряженных НЧ молекулы МГ располагаются дальше, следовательно, падает эффективность безызлучательной дезактивации. Однако, за счет меньшей локальности, на данных расстояниях проявляется усиление индуцированного поглощения в полосе $S_{0} \rightarrow S_{1}$-перехода, что приводит к увеличению насыщения поглощения раствора и усилению сигнала Z-сканирования в перетяжке пучка (рис. 8,a). Это подтверждается фотометрическими измерениями, которые показывают, что при неизменой концентрации красителя введение в раствор плазмонных НЧ независимо от их знака заряда увеличивает оптическую плотность растворов.
В этанольных растворах красителя наблюдается нелинейное усиление поглощения, связанное с накоплением молекул в триплетном возбужденном состоянии. Поэтому наибольшее влияние на нелинейное поглощение МГ оказывают отрицательно заряженные НЧ, притягивающие краситель на дистанцию эффективного безызлучательного тушения, что приводит к ускоренной дезактивации и уменьшению населенностей возбужденных состояний красителя. Для положительно заряженных НЧ данный эффект выражен существенно слабее.

\section{Заключение}

Методом Z-сканирования определены диапазоны концентраций растворов МГ, в которых оптическая нелинейность растворов определяется димерной и мономерной формами красителя, и предложена качественная модель для описания данного механизма нелинейности.

Показано, что оптическая нелинейность разбавленных растворов красителя обнаруживает сильную зависимость от вида растворителя. В водном растворе имеет место некогерентное насыщение поглощения в переходе между $S_{0}$ - и $S_{1}$-состояниями, а в спиртовых - нелинейное усиление поглощения в полосе перехода $T_{1} \rightarrow T_{2}$, связанное с увеличением времени жизни триплетных возбужденных состояний молекул.

Выявлена зависимость амплитуды нелинейного отклика растворов красителя от концентрации плазмонных НЧ и знака их поверхностного заряда. В водных растворах МГ добавление НЧ, имеющих отрицательный поверхностный заряд, приводит к уменышению нелинейного насыщения поглощения красителя. Положительно заряженные НЧ увеличивают амплитуду нелинейного оптического просветления раствора практически вдвое. В спиртовых растворах внесение отрицательно заряженных НЧ приводит к уменьшению нелинейного поглощения красителя примерно в 3 раза.

\section{Конфликт интересов}

Авторы заявляют, что у них нет конфликта интересов.

\section{Список литературы}

[1] Kang I., Krauss T., Wise F. // Opt. Lett. 1997. V. 22. P. 1077. doi 10.1364/OL.22.001077.

[2] Semashko V.V., Akhtyamov O.R., Shavelev A.A. Rakhimov N.F., Lovchev A.V. // Laser Phys. Lett. 2018. V. 15. P. 035702. doi 10.1088/1612-202X/aa9c04.

[3] Stryland E.W., Sheik-Bahae M.Z. // Charact. Tech. Tabul. Org. Nonlinear Mater. 1998. N 3. P. 655.

[4] Ганеев Р.А., Усманов Т.Б. // Квант. электр. 2007. Т. 37. N 7. C. 605; Ganeev R.A., Usmanov T. // Quant. Electron. 2007. V. 37. N 7. P. 605. doi 10.1070/QE2007v037n07ABEH013367. 
[5] Tardivo J.P., Giglio A.D., Oliveir C.S. et al. // Photodiagn. Photodyn. Ther. 2005. N 2. P. 175-91. doi 10.1016/S15721000(05)00097-9.

[6] Sadigh M.K., Zakerhamidi M.S., Rezaei B., Milanchian K. // J. Mol. Liq. 2017. P. 548. doi 1016/j.molliq.2016.12.108.

[7] Ganeev R.A., Zvyagin A.I., Ovchinnikov O.V., Smirnov M.S. // Dyes and Pigments. 2018. V. 149. P. 236. doi 10.1016/j.dyepig.2017.09.063.

[8] Li X., Chen Y., Huang X. // J. Inorg. Biochem. 2007. V. 101. P. 918. doi 10.1016/j.jinorgbio.2007.03.001.

[9] Frens G. // Nat. Phys. Sci. 1973. V. 241. P. 20. doi 10.1038/physci241020a0.

[10] Li Z.P., Duan X.R., Liu C.H., Du B.N. // Anal. Biochem. 2006. V. 351. P. 18. doi 10.1016/j.ab.2006.01.038.

[11] Andreu-Navarro A., Fernandez-Romero J.M., GomezHens A. // Anal. Chim. Acta. 2012. V. 713. P. 1. doi 10.1016/j.aca.2011.11.049.

[12] Veselkov A.N. Davies D.B. Sev.: SevNTU Press. 2002. 260 p.

[13] Кучеренко М.Г., Русинов А.П. // Квант. электр. 2004. Т. 34. № 8. C. 779; Kucherenko M.G. Rusinov A.P. // Quant. Electron. 2004. V. 34. N 8. P. 779. doi 10.1070/QE2004v034n08ABEH002848.

[14] Климов В.В. Наноплазмоника. М.: Физматлит., 2009. 480 с.

[15] Русинов А.П., Кучеренко М.Г. // Вестник ОГУ. 2015. Т. 188. № 13. C. 195.

[16] Кучеренко М.Г. // Вестник ОГУ. 2012. Т. 137. № 1. С. 141. 